\title{
Surgical Treatment of an Osteopetrotic Patient with Postoperative Fractures: Lessons from Siblings with Osteopetrosis
}

\author{
Tao Huang, ${ }^{1}$ Qingwei Liang, ${ }^{1}$ Hao Qian, ${ }^{1}$ Xiucheng $\mathrm{Li}^{1}$ and Chunping Zou ${ }^{1}$ \\ ${ }^{1}$ Department of Orthopedics, First Affiliated Hospital of China Medical University, Shenyang, Liaoning, P.R. \\ China
}

\begin{abstract}
Osteopetrosis is a rare, inherited disease characterized by dense and brittle bones, and it is associated with an increased risk of femoral fractures. However, the surgical treatment of the fracture patients with osteopetrosis is a subject of controversy, because it is difficult to compare the treatment effects between surgical treatment and conservative treatment in part due to the rarity of siblings with osteopetrosis. In fact, the genetic background and the environmental factors, such as living conditions and daily work, may influence the prognosis of the fracture patients with osteopetrosis. Here we describe siblings with osteopetrosis, a 23-year-old female patient and her older brother, both of whom had suffered from bilateral femoral fractures at their childhood. They do not have other brothers or sisters, and their parents are healthy. The younger sister had undergone surgical treatment for femoral fractures at the age of 8 years and recovered very well. In contrast, her brother had received the conservative treatment for femoral fractures at the age of 6 years, and thereafter he is badly disabled due to the malunion of the fractured femurs. Apparently, the surgical treatment is superior to the conservative treatment for the fracture patient with osteopetrosis. Unfortunately, fifteen years later, the sister fractured again at both femurs and underwent another operation. She recovered quickly from the second operation, indicating that second operation should be considered in osteopetrosis patients with postoperative fractures. The present report provides valuable information for the choice of treatment of femoral fractures associated with osteopetrosis.
\end{abstract}

Keywords: conservative treatment; fracture; internal fixation; osteopetrosis; surgery

Tohoku J. Exp. Med., 2013 June, 230 (2), 93-96. C 2013 Tohoku University Medical Press

\section{Introduction}

Osteopetrosis is a rare disorder of bone development, which was first found by Albers-Schonberg, also known as the marble bone or Albers-Schonberg disease (Armstrong et al. 1999). The disease is characterized by extensive bone sclerosis and closed medullary cavity (Bhargava et al. 2009). The fragility of such dense bone induced by bone sclerosis may increase the incidence of fractures (Armstrong et al. 1999; Fotiadou et al. 2009). The fractures most frequently involve the upper third of the femur and the upper third of the tibia (Golden and Rodriguez 2010). The internal fixation is difficult to preform in part due to bone fragility; therefore, orthopedists may prefer to conservative treatment with skeletal traction or plaster fixation. Up to now, there have been only limited reports that compared the effectiveness of the treatment for fractured patients with osteopetrosis between conservative and operation treatments (Landa et al. 2007; Rafiq et al. 2009). However, these reports should be evaluated with caution, because patients from these reports have the different genetic back- ground and life style, each of which may influence the treatment effect.

In the present report, we describe the siblings with osteopetrosis, a brother and a sister, both of whom had suffered from the bilateral femoral fractures and grew up in the same family. The older brother was given conservative treatment and the younger sister underwent internal fixation operation. Thus, we are able to compare the treatment effects between conservative treatment and surgical treatment. We also describe the successful surgical treatment of the sister with postoperative fractures.

\section{Clinical Report}

\section{Basic clinical information}

The patient is a 23 -year-old female who was sent to the emergency department immediately after she fell down the stairs. She complained of pain in both lower limbs and was unable to walk. The patient had a known history of osteopetrosis and had previously suffered bilateral femoral fractures, which had been managed with surgical treatment when she was eight years old. Her parents have two chil-

Received April 24, 2013; revised and accepted May 27, 2013. Published online June 14, 2013; doi: 10.1620/tjem.230.93.

Correspondence: Tao Huang, First Affiliated Hospital of China Medical University No155, Nanjing Street, Heping District, Shenyang, Liaoning 110001, P.R. China.

e-mail: huangtao@mail.cmu.edu.cn 


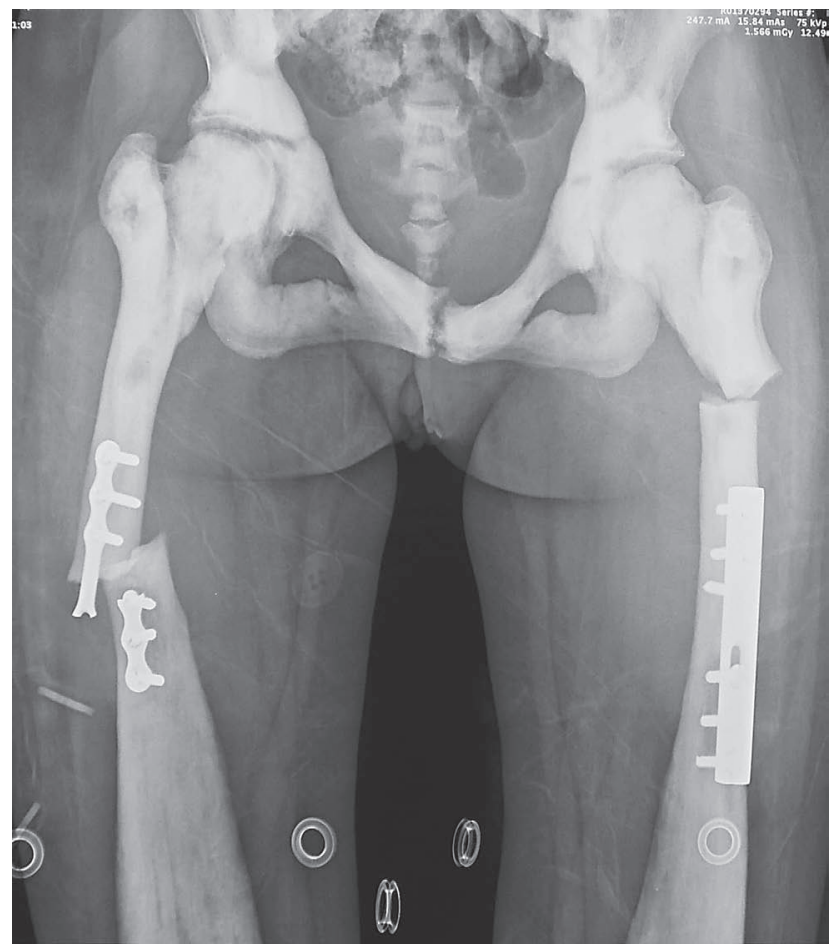

Fig. 1. X-ray of bilateral femurs of the female patient with osteopetrosis before the second operation. The fracture of the bilateral femur implanted with plate is obvious, and the plate in the right femur was broken.

dren, and her older brother is also an osteopetrosis patient, who had bilateral femoral fractures at the age of 6 . Unfortunately, he has been seriously disabled, because no surgical treatment was given to him, resulting in fracture malunion. Her parents and other relatives are not osteopetrosis patients.

Physical examinations revealed the bilateral pathologic movement and swelling at both femurs. All distal artery pulses were palpable. The radiographs showed increased bone density and bilateral femoral fractures that had been fixed with plates (Fig. 1). It should be noted that the right plate was broken. The osteopetrotic images of the chest and humerus showed the excessive calcification of the bone (Fig. 2). Her anemia was obvious; RBC was $3.04 \times 10^{12}$ and $\mathrm{Hb}$ was $84 \mathrm{~g} / \mathrm{L}$. All the above clinical manifestations proved the diagnosis of osteopetrosis. Due to the second fractures after internal fixation, the operation risk is very large. However, she was eager to have operation, because she did not want to be someone like her brother who had been seriously disabled because of fracture malunion due to the conservative treatment. According to her explanation, she had recovered her walking function after the first internal fixation operation. Therefore, she craved for another internal fixation on both broken legs.

\section{The treatment experience and clinical effects}

After a full research and discussion, we decided to give the patient surgical treatment. Before the internal fixa-

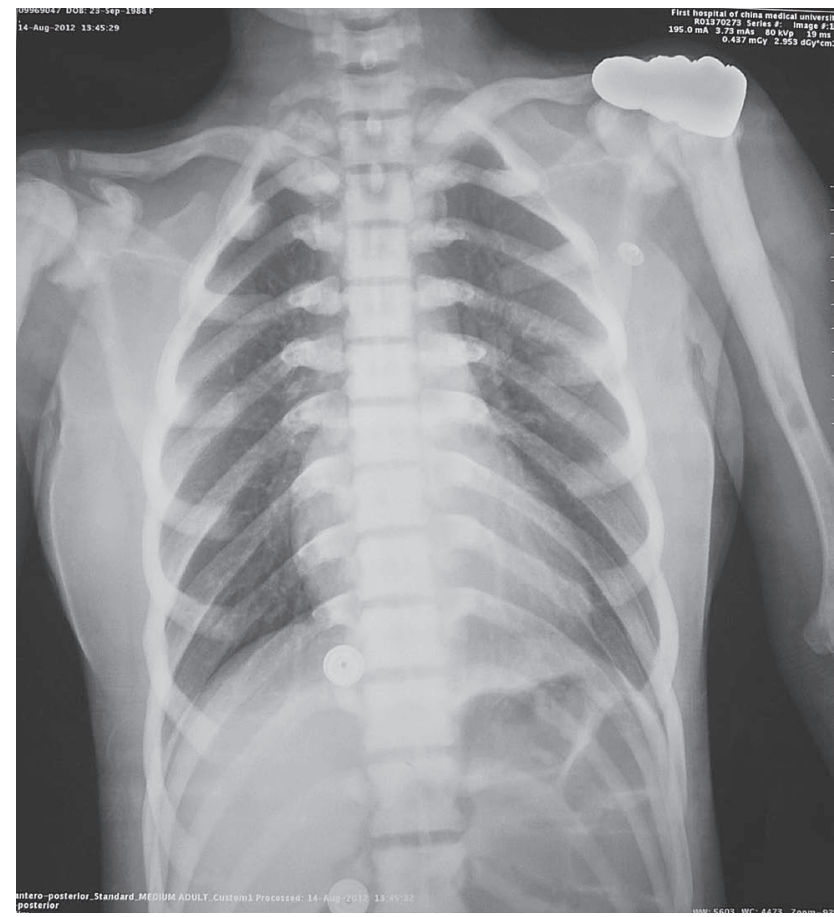

Fig. 2. Chest X-ray image of the female osteopetrosis patient. Note the calcified bone and closed marrow cavity in the ribs, vertebrae and humerus.

tion, we had to remove the plates installed last time. For the right leg, the broken plate was removed with great efforts (Fig. 1). However, it was difficult to remove the plate from left femur because the shaft near the plate was surrounded by the structure like reinforced concrete. Then, we decided to leave the left plate. During the operation we found that there was no obvious medullary cavity at all and the bone was too hard to penetrate by drilling. In order to avoid the breaking of a bit, we had to drill holes very slowly and change bits frequently. Finally, we successfully performed the internal fixation on both femurs with plates (Fig. $3 a, b)$. The patient had recovered well and began to have limb exercise before leaving hospital. No complications appeared after the operation.

In order to compare the treatment effect between the surgical treatment and conservative treatment, we obtained her brother's X-ray images. The brother also had bilateral femoral fractures at his childhood (Fig. 4a). However, he was only given the skeletal traction, and he did not recover as good as his sister. His X-ray imaging shows the fracture malunion (Fig. 4b).

\section{Discussion}

Osteopetrosis is a metabolic disorder with diminished bone resorption due to osteoclastic abnormality (Bhargava et al. 2009). Its main pathological changes are excessive calcification of osteoid tissue. Clinically, the fragility of dense, sclerotic bones often leads to an increased incidence of fractures (Ashby 1992; Benum et al. 2010). In the present report, we are able to conclude the following points. 


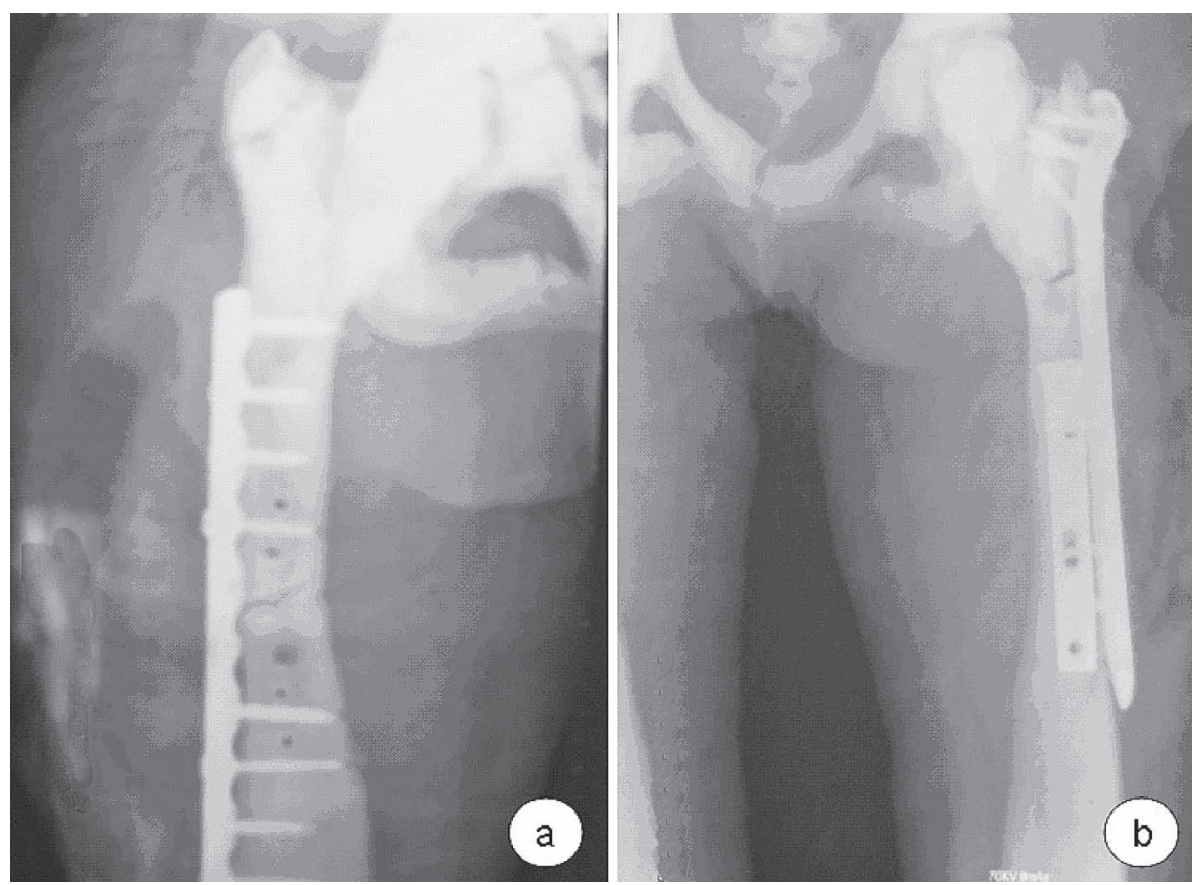

Fig. 3. X-ray of bilateral femurs of the female patient after the second operation.

Fracture was corrected with internal fixation, and bilateral femurs were implanted with new plates. The old plate was removed from the right femur (a), but was not removed from the left femur (b).

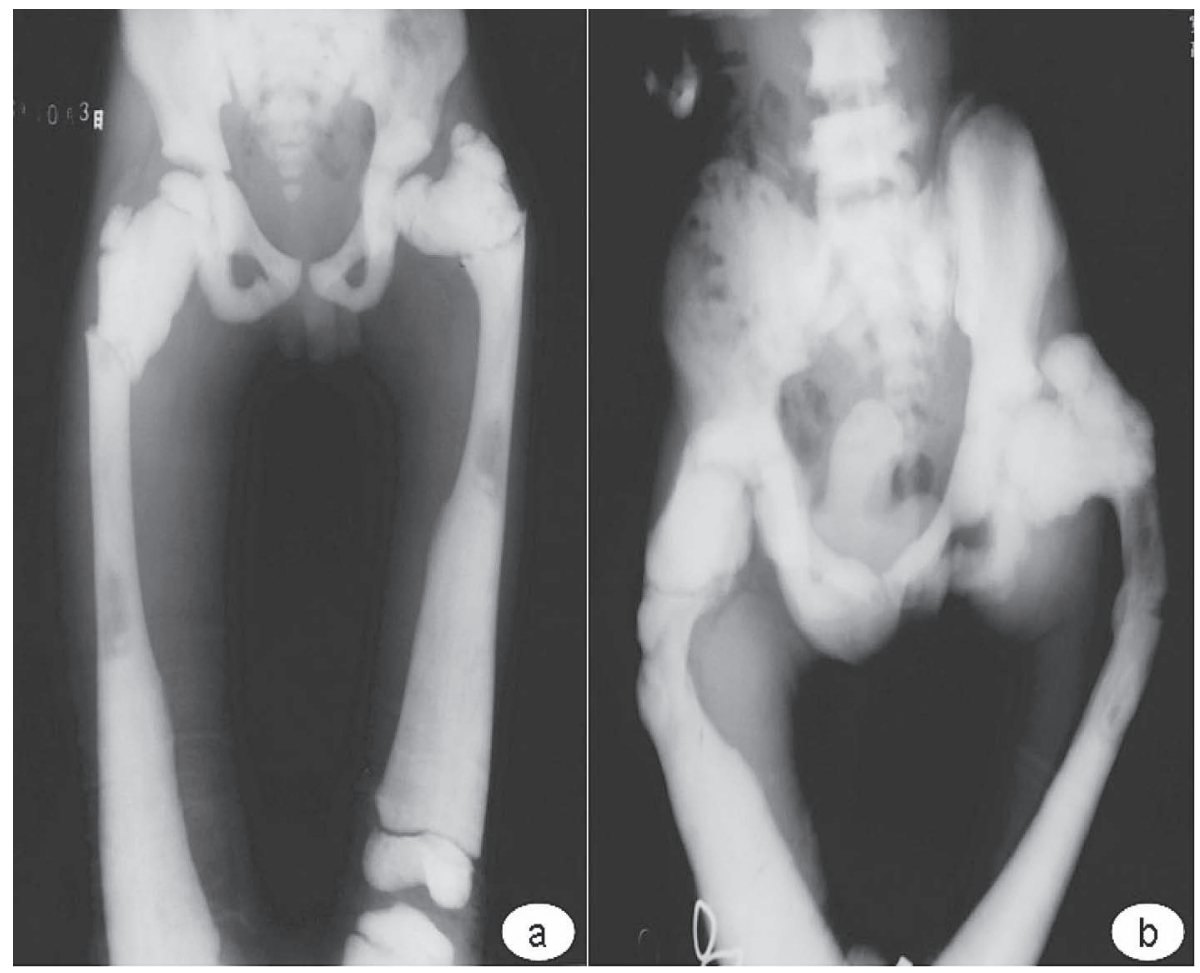

Fig. 4. X-ray of bilateral femurs of the patient's brother.

The deformity of the bilateral femurs fracture was not obvious (a), but the malunion and deformity of the bilateral femurs are very serious after the conservative treatment three years later. 
Firstly, osteopetrosis does really have a family tendency and has nothing to do with gender. The brother and sister's fractures all occurred at the similar positions of the femurs. Osteopetrosis can be divided into two types: mild type and severe type. The mild type shows dominant heredity and the severe type shows recessive heredity. Since both of the patients have severe symptoms and all the relatives are not osteopetrosis patients, the two patients got disease by the way of recessive heredity. Secondly, the consequences of conservative treatment and operative treatment after femoral fractures are entirely different (Figs. 3 and 4). The surgical treatment is obviously superior to the conservative treatment, although the occlusive intramedullary cavity and hard brittle bone greatly increased the risk of surgery, such as intraoperative fracture and osteonecrosis. We would like to emphasize that young patients with good general conditions should take the operation to improve the prognosis and quality of life in order to reduce fracture malunion. Because the bone of patients with osteopetrosis is hard and brittle, it is very difficult for surgeons to drill the bone or fix a plate along the bone. It should be noted that too much strength may cause a new fracture (Chhabra et al. 2005; Landa et al. 2007).

The surgeon will face two challenges in the treatment of osteopetrosis patients with recurrent fractures; the first is how to remove the implanted steel and the second is how to install a new plate onto the bone (Ramiah et al. 2006). For the first challenge, we should anticipate unsuccessful removal of steel plate before operation. In our case, the plate on the right leg was removed smoothly, whereas we failed to remove the plate from left femur because the shaft near the plate was surrounded by the structure like reinforced concrete. However, it is not necessary to remove all the implants from the bone (see Fig. 3b); therefore, the surgeons should be flexible when facing the first challenge. For the second challenge, extreme care and caution are required for drilling, reaming, or inserting implants in patients with osteopetrosis to prevent a bit-breaking, such as proper strength and no shaking hands while driving the drill bit. Considering patients' quality of life in the future, surgeons should make great efforts to take operative treatment as much as possible. The success of the treatment lies in suitable selection of internal fixation and caution during surgery (Rafiq et al. 2009; Wang et al. 2010).
Because osteopetrosis is a very rare disease, it is almost impossible to study a large number of patients, especially siblings with the same genetic background and life style. In the present report, by comparing the siblings with osteopetrosis, we propose that the operation with the internal fixation devices is superior to the traditional conservative treatment for the fractures. Furthermore, the present report provides supportive information for the surgical treatment of femoral fractures associated with osteopetrosis.

\section{Conflict of Interest}

The authors have no conflict of interest.

\section{References}

Armstrong, D.G., Newfield, J.T. \& Gillespie, R. (1999) Orthopedic management of osteopetrosis: results of the survey and review of the literature. J. Pediatr. Orthop., 19, 122-132.

Ashby, M.E. (1992) Total hip arthroplasty in osteopetrosis: a report of two cases. Clin. Orthop. Relat. Res., 276, 214-221.

Benum, P., Aamodt, A. \& Nordsletten, L. (2010) Customised femoral stems in osteopetrosis and the development of a guiding system for the preparation of an intramedullary cavity: a report of two cases. J. Bone Joint Surg. Br., 92, 1303-1305.

Bhargava, A., Vagela, M. \& Lennox, C.M. (2009) "Challenges in the management of fractures in osteopetrosis" Review of literature and technical tips learned from long-term management of seven patients. Injury, 40, 1167-1171.

Chhabra, A., Westerlund, L.E. \& Kline, A.J. (2005) Management of proximal femoral shaft fractures in osteopetrosis: a case series using internal fixation. Orthopedics, 28, 587-592.

Fotiadou, A., Arvaniti, M. \& Kiriakou, V. (2009) Type II autosomal dominant osteopetrosis: radiological features in two families containing five members with asymptomatic and uncomplicated disease. Skeletal Radiol., 38, 1015-1021.

Golden, R.D. \& Rodriguez, E.K. (2010) Management of bilateral subtrochanteric femur fractures with internal fixation and rhbmp-7 in a patient with osteopetrosis. J. Med. Case Rep., 4, 142-146.

Landa, J., Margolis, N. \& Di Cesare, P. (2007) Orthopaedic management of the patient with osteopetrosis. J. Am. Acad. Orthop. Surg., 15, 654-662.

Rafiq, I., Kapoor, A. \& Burton, D.J. (2009) A new modality of treatment for non-united fracture of the humerus in a patient with osteopetrosis: a case report. J. Med. Case Rep., 13, 15-17.

Ramiah, R.D., Baker, R.P. \& Bannister, G.C. (2006) Conversion of failed proximal femoral internal fixation to total hip arthroplasty in osteopetrotic bone. J. Arthroplasty, 21, 1200-1202.

Wang, J., Liang, Y. \& Zhang, Q. (2010) Total joint arthroplasty in a patient with osteopetrosis: 10-year follow-up. Orthopedics, 16, 264-266. 